\title{
Die Structur der electrischen Platten von Torpedo.
}

\author{
Von
}

\section{Dr. Franz Boll,}

Privatdocenten u. Assistenten am physiolog. Laboratorium der UniversitätBerlin.

\section{Hierzu Tafel VIII.}

Seitdem durch die Entdeckung der electrischen Platte der Nachweis einer allen electrischen Organen gemeinsamen morphologischen Einheit geführt worden war, sind unsere Kenntnisse über den Bau dieser räthselhaften Organe so gut wie stationär geblieben. Noch heute repräsentirt die abschliessende Monographie Max Schultze's ${ }^{1}$ ) den Standpunkt unseres Wissens. Ein halbes Menschenalter lang hat die anatomische Forschung dieser Darstellung keine neue Thatsache hinzuzufügen vermocht.

Die seither erschienenen Arbeiten uber den Bau des electrischen Organs, so weit mir bekannt nur drei an Zahl, beziehen sich einzig und allein auf Torpedo.

Von diesen beschäftigt sich die Mittheilung Babuchin's ${ }^{2}$ ) allein mit der Histiogenese der electrischen Organe, ohne über die Structur derselben speciell über die feinere Anatomie der electrischen Platten etwas Neues beizubringen.

1) Zur Kenntniss der electrischen Organe der Fische. Erste Abtheilung Malapterurus, Gymnotus. Abhandlg. der Naturforschenden Gesellsch. in Halle. Bd. IV, 1858. Zweite Abthlg. Torpedo. Ebend. Bd. V, 1859.

2) Entwickelung der electrischen Organe und Bedeutung der motorischen Endplatten. Centralblatt f. d. med. Wissenschaft. 1870, S. 241. 257. 
Die Mittheilung Ciaccio's ${ }^{1}$ ), welche sich ebenso wie die von Babuchin als eine vorläufige ankündigt, hat speciell die Nervenendigungen im electrischen Organ zu ihrem Gegenstande. Auch Ciaccio lässt, -wie Max Schultze, die Nerven in dem von Koelliker ${ }^{2}$ ) entdeckten, die Bauchfläche der electrischen Platte überziehenden feinsten Terminalnetz markloser Nervenfasern, welches ihm durch Goldchlorid zu färben gelang, endigen. Im Ganzen bietet seine Darstellung nur unerhebliche Abweichungen von der Max Schultze's.

Auch der neueste Untersucher der electrischen Organe, de Sanctis ${ }^{3}$ ), der sich wie Babuchin gleichfalls viel eingehender als mit der Structur mit der Entwickelungsgeschichte der electrischen Organe beschäftigt, bleibt in der Frage von der feineren Structur der electrischen Platten bei dem Terminalnetze Ko elliker's stehen. Einige Zusätze, welche er zu der Lehre von der Nervenendigung, wie dieselbe durch Max Schultze festgestellt ist, zu machen sich gedrungen fühlt, beruhen auf offenbarem Irrthum, wie schon ein Blick auf die von de Sanctis selbst gegebenen phantasiereichen Abbildungen lehrt. Es sollen blasse Nervenfasern mit den Nerven der electrischen Platten in Verbindung stehen und so eine Art keulenoder knopfförmiger Endigung darstellen, die Kerne nicht nur derselben, sondern auch benachbarter Platten sollen durch blasse Nervenfasern netzartig mit einander verbunden sein $u$. s. w.

Es ist mir im Frühlinge d. J. während eines Aufenthaltes in Viareggio gelungen, die Erkenntniss der feineren Structur der electrischen Platten über das von Koelliker entdeckte und von Max

1) Intorno al finale distribuimento di nervi nell' organo elettrico della Torpedine (Torpedo narke Rosso). S. Richiardi e G. Canestrini Archivio per la Zoologia, l'Anatomia e la Fisiologia, Serie II, Vol. II, Fascicolo I, S. 1, 1870. Vergl. über diese den deutschen Fachgenossen schwer zugängliehe Arbeit mein Referat im Centralblatt f. d. med. Wissenschaft 1873.

2) Untersuchungen zur vergleichenden Gewebelehre. Verhandlg. der physiol. med: Gesellschaft zu Würzburg 1856.

3) Embriogenia degli organi elettrici delle torpedini e degli organi pseudo-elettrici delle. Raje. Atti della. R. Academia delle Scienze Fisiche e Matematiche di Napoli V. 1872. Vergl. mein Referat im Centralblatt f. d. med. Wissenschaft. 1873. 
Schultze genauer untersuchte Terminalnetz hinaus noch einen Schritt weiter zu führen und ein Structurverhältniss aufzufinden, welches noch feiner ist und, un gesehen zu werden, noch vervollkommneterer optischer Hülfsmittel bedarf, wie das Terminalnetz Koelliker's, welches zu der Zeit seiner Entdeckung, 1856, als an der Grenze des Sichtbaren und des Leistungsvermögens der Mikroskopie betrachtet werden durfte. Jetzt wird das Terminalnetz bereits durch Linsen, wie Hartnack's VII (allerdings nur in besonders günstigen Fällen) erkannt und bei Hartnack's Nr. VIII bereits deutlich als Netz aufgelöst; bei der Linse Nr. IX (älterer Construction) wird man stets auf eine völlig befriedigende Darstellung des Terminalnetzes mit Sicherheit rechnen können. Aber diese Linsensysteme sind noch ungenügend, die feine Structur wahrzunehmen, um die es sich hier handelt. Man wird zum Studium dieser Verhältnisse die $\mathrm{H}$ a $\mathrm{r} \mathrm{t} \mathbf{\mathrm { n }}$ ack' $\mathrm{k}$ chen Immersionslinsen nicht entbehren können. Die Systeme IX à immersion und $\mathrm{X}$ à immersion lassen die fragliche Structur bereits deutlich erkennen. Immerhin ist es nicht ganz leicht, selbst bei diesen Vergrösserungen die erste Erkenntniss der Structur zu gewinnen. Ich selber hatte schon mehrere Tage lang die electrischen Platten mit der Immersionslinse IX untersucht, ohne auf diese feine Structur, die sich mit ausserordentlicher Regelmässigkeit über die ganze Fläche der Platte erstreckt, aufmerksam zu werden, bis sich mir die Wahrnehmung derselben ganz plötzlich einmal aufdrängte. Auch habe ich später noch die Erfahrung gemacht, wie schwer es ist, anderen, denen ich meine Präparate demonstrirte, diese Structur zur Anschauung zu bringen, und dass es gewöhnlich längere Zeit dauert und einer längeren Uebung und Anstrengung bedarf, dieser Structur überhaupt optisch habhaft zu werden. Doch ist es auch in diesem Falle nur der erste Schritt, welcher schwer fällt. Hat man überhaupt die Structur, um die es sich hier handelt, zum ersten Male erfasst, so gelingt es dann leicht, sie stets und unter allen, auch ungünstigeren Verhältnissen, schlechterer Beleuchtung, ungenügenderer Conservirung u. s. w., zu sehen und zu studiren. Ja, man wird finden, dass die Immersionslinse IX zum Studium der fraglichen Verhältnisse eigentlich vollständig ausreicht und dass durch stärkere Linsen, z.B. X, kaum erheblich bessere Bilder, geschweige denn tiefere Einsichten in das fragliche Structurverhältniss gewonnen werden. Es gelingt mitunter sogar unter besonders günstigen Verhältnissen bereits mit der trocknen 
Linse IX von Hartnack diese Structurverhältnisse zu erkennen, jedoch nur dann, wenn man vorher durch Immersionslinsen das Bild genau kennen gelernt hat und weiss, was man zu suchen hat. Doch halte ich es für unmöglich, Jemand, der bisher die Structur mit Immersionslinsen nicht gesehen hat, durch diese Linse allein eine Anschauung davon zu verschaffen.

So viel von den optischen Bedingungen, unter denen es allein möglich ist, diese Structur zu erkennen. Es erübrigt noch, von der Methode der Untersuchung und der Herstellung der Präparate zu reden.

Ich kenne nur zwei Methoden, unter denen diese Structur in wirklich befriedigender Weise wahrgenommen werden kann: Die Untersuchung im frischen Zustande und die Behandlung mit Ueberosmiumsäure. Die Untersuchung im frischen Zustande geschieht am Besten nach der von Max Schultze angegebenen Präparationsmethode in einem Tropfen Liquor cerebrospinalis. Die Ueberosmiumsäure habe ich stets nur in einprocentiger Lösung angewandt, indem ich etwa erbsengrosse Stücke des absolut frischen Organs längere oder kürzere Zeit in grösseren oder kleineren Mengen der Flüssigkeit verweilen liess. Nachdem die Stückchen intensiv schwarz gefärbt waren, nahm ich sie aus der Osmiumsäure und that sie entweder sogleich oder nach einem ein- bis mehrtägigen Aufenthalt in destillirtem Wasser in die von Max Schultze empfohlene concentrirte wässerige Lösung des essigsauren Kali, in welcher die Stücke unbeschränkte Zeit verweilen können, ohne irgendwelche Veränderungen zu erleiden. An allen den so behandelten Präparaten ist die von mir entdeckte Structur ausnahmslos gut erhalten, - an einigen allerdings schärfer und klarer, an anderen weniger gut und deutlich. Im Allgemeinen sind jedoch diese Unterschiede sehr gering. Dennoch sind die so erhaltenen Osmiumpräparate von sehr verschiedener Güte, wenn auch aus einem anderen Grunde und nach einer anderen Hinsicht, wie nach der Demonstration der Structur der electrischen Platte: Es gibt nämlich Osmiumpräparate, an denen das Koelliker'sche Terminalnetz schlecht oder so gut wie gar nicht $\mathrm{zu}$ sehen ist und. solche, an denen dasselbe in vollkommener Deutlichkeit conservirt ist. An beiden Arten von Präparaten ist die unten $z u$ beschreibende Structur der electrischen Platten in vollkommener identischer Deutlichkeit erhalten. Die wichtigen und interessanten Beziehungen der Structur $\mathrm{zu}$ dem 
Terminalnetz, die derselben erst ihre wahre Bedeutung verleihen, können natürlich nur an denjenigen Osmiumpräparaten mit Erfolg studirt werden, an denen auch das Terminalnetz deutlich zu sehen ist. Das richtige Verständniss des Zusammenhanges beider Bildungen, des Koelliker'schen Netzes und meiner Structur, die sich gegenseitig auf das Intimste bedingen, wird eben nur an solchen Präparaten möglich sein, in denen beide Bildungen in gleicher Deutlichkeit zu sehen sind.

Ich habe vergebens die Bedingungen zu ermitteln getrachtet, unter denen man mit Sicherheit neben der vollkommenen Darstellung meiner Structur, auf die man, wie gesagt, bei Osmiumpräparaten fast stets rechnen kann, eine ebenso vollkommene Darstellung des Terminalnetzes erhalten würde. Thatsache ist, dass unter 15 verschiedenen Stückchen electrischer Organe, die ich von Viareggio in einem Fläschchen essigsauren Kali mit nach Berlin gebracht habe, und die alle mit einprocentiger Osmiumsäure, sonst aber in Bezug auf Zeit der Einwirkung, Flüssigkeitsmenge, längeren oder kürzeren, resp. gar keinen Aufenthalt im destillirten Wasser alle verschieden behandelt waren, etwa die Hälfte nur meine Structur und von dem Terminalnetz nur ungenügende Spuren, die andere Hälfte neben meiner Structur auch mehr oder minder befriedigend das Terminalnetz zeigt. Auch in Viareggio selber wollte es mir nicht gelingen, eine bestimmte Behandlungsmethode zu finden, welche eine Darstellung des Terminalnetzes in vollkommener Weise garantirt hätte. Im Allgemeinen erhielt ich bessere Resultate, wenn ich die geschwärzten Stückchen nur ganz kurze Zeit oder auch gar nicht in destillirtem Wasser auswusch, sondern dieselben alsbald in die Lösung des essigsauren Kali brachte.

Alle anderen Methoden, die ich sonst versucht habe, (Maceration in den bekannten Max Schultze'schen dünnen Chromsäurelösungen, Glycerin, Alkohol, Pikrokarmin) erwiesen sich als absolut ungenügend, meine Structur wie das Koelliker'sche Terminalnetz zu conserviren. Die besten Resultate ergab mir noch die Müller'sche Flüssigkeit. Stücke electrischer Organe, die ich in Müller'scher Flüssigkeit von Viareggio nach Berlin gebracht hatte, lassen noch jetzt (nach zwei Monaten) die Punktirung der electrischen Platten ziemlich deutlich erkennen, wenn auch das Bild mit den Osmiumpräparaten freilich nicht verglichen werden darf. Von dem Terminalnetz 
ist an diesen Präparaten keine Spur zu sehen. Das von Ciaccio zu der Darstellung des letzteren mit solchem Erfolge angewandte Goldchlorid habe ich leider nicht versuchen können.

Bei der Darstellung meiner Untersuchungen über die Structur der electrischen Platten und die Nervenendigung in derselben kann ich in vielen Punkten um so kürzer sein, als eine Reihe der wesentlichsten anatomischen Thatsachen, die bis dahin noch streitig waren, durch die Monographie Max Schultze's in derartiger Weise hors de discussion gesetzt sind, dass es genügt, einfach auf diese Darstellung zurückzuverweisen. Nur zur Orientirung und zur Begränzung der Frage, um die es sich hier handelt, werde ich die Figg. 1 u. 2 erläutern, von denen ich nur hervorheben will, dass sie bis auf das klẹinste Detail mit grösstmöglichster Treue je einem einzigen Präparate nachgebildet und nicht als das Resultat schematisirender $\mathrm{Zu}$ sammensetzung anzusehen sind.

Fig. 1 ist bei einer Vergrösserung von IV, $3 \mathrm{Hartnack}$ gezeichnet worden und stellt das Verästelungsgebiet einer einzigen markhaltigen Nervenprimitivfaser dar, die ich - da in der Literatur über die electrischen Organe eine passende Bezeichnung für diese Fasern bisher nicht vorliegt, - als Nervenfasern erster Ordnung bezeichnen will. Diese Nervenfasern erster Ordnung gehen am Rande der electrischen Platten, wie sie zuerst Rud. Wagn er beschrieben und abgebildet hat, aus breiten, einfachen, mit sehr dicker Schwannscher Scheide versehenen Nervenfasern (die ich Stammfasern nennen will) hervor, die auf einmal in 10-20 und noch mehr Aeste ausstrahlen. Diese Aeste (die Nervenfasern erster Ordnung) verlaufen alle sehr vielfach sich dichotomisch theilend von ihrer Ursprungsstelle am Rande der electrischen Platten aus über die ventrale Fläche der Platten hinweg, bis endlich ihre letzten, gestreckt verlaufenden Endäste in der in Fig. 1 gezeichneten Weise in eine Mehrzahl (hier fünf) kurze Fasern übergehen, die ich als die Nervenfasern zweiter Ordnung bezeichnen will. Diese Nervenfasern zweiter Ordnung bilden nach nur sehr kurzem Verlauf eine sehr charakteristische Verästelung, die grosse Aehnlichkeit mit der Verzweigung eines Hirschgeweihes hat. Bei einer stärkeren Vergrösserung (Hartnack's IX sec., 2) ist in Fig. 2 das totale Verästelungsgebiet einer solchen Nervenfaser zweiter Ordnung wiedergegeben. 
Auf beiden Abbildungen sieht man eine Anzahl von Kernen in unregelmässiger Weise über die Fläche der Platte, die stets als von der Bauchfläche her betrachtet $\mathrm{zu}$ denken ist ${ }^{1}$ ), zerstreut. Schon an der bei schwacher Vergrösserung gezeichneten Fig. 1 unterscheidet man deutlich zwei Arten derselben, ovale Kerne, die sich dem Verlauf der Nervenverästelung anschliessen, und runde, sehr selten ellipsoidische Kerne, die abseits von den Nervenfasern frei in der Fläche des Gesichtsfeldes liegen. Eine sorgfältige Einstellung ergibt, dass die ersteren in der That auch in der Fläche der Nervenverästelung, d. h. wirklich über der Bauchfläche der Platte liegen, während die letzteren stets tiefer und ausnahmslos in der Substanz der Platten selbst eingebettet sind.

lit stärkeren Vergrösserungen untersuchend erkennt man, dass die ovalen dem Verlauf der Nervenverästelung sich anschmiegenden Kerne, wie M. Schultze bereits ausführlich beschrieben und abgebildet hat, zweierlei Formenkreisen angehören. An den Nervenfasern erster Ordnung sind es einfache Kerne, die in der Substanz der weiten, die Nervenfaser wie ein schlotteriger Sack umgebenden Schwannschen Scheide gelegen sind - und zwar dicht hintereinander, so dass sie mitunter eine fast continuirliche Reihe bilden. An den beiden Nervenfasern zweiter Ordnung, welche einer Schwann'schen Scheide entbehren, sind es wirkliche Bindegewebszellen, den Sternzellen des die Zwischenräume zwischen den electrischen Platten ausfüllenden Gallertgewebes gleichend, die in der in Fig. 2 dargestellten Weise mit ihren ovalen Kernen und dem nur sehr wenig entwickelten spindelförmigen Zellenleibe der Längsaxe der Nervenfasern parallel anliegen und auch oft ihre langen faserartigen Ausläufer dem Verlauf der Ner'venverästelung anschmiegen.

In Bezug auf die runden frei in der Fläche des Gesichtsfeldes liegenden Kerne muss ich mit Max Schultze gegen Koelliker betonen, dass dieselben ausnahmslos in der Substanz der Platten selber liegen und niemals auf der freien Oberfläche derselben vor-

1) Hieraus ergiebt sich, dass die im Laufe dieser Darstellung häufig wiederkehrenden Bezeichnungen ,oben" und ,unten" nicht so aufzufassen sind, als ob ich mir die Torpedo auf dem Rücken schwimmend vorstellte. Vielmehr beziehen sich diese Bezeichnungen nur auf die einzelne electrische Platte, die am besten und bequemsten von der Seite der Nervenausbreitung, $d . h$. von der BauchHläche her im mikroskopischen Bilde betrachtet wird. 
kommen. Dieselben sind stets deutlich doppeltcontourirt und gleichen vollkommen den (gleichfalls doppeltcontourirten) Kernen, die in der grauen molecularen Masse der Hirnrinde (Neuroglia Virchow's) eingebettet liegen. Nicht selten (besonders deutlich an conservirten Präparaten) sieht man sie von einem hellen, scharfbegränzten rundlichen Raum umgeben, dem Ausdruck eines schwächeren Lichtbrechungsvermögens der benachbarten Zone der Grundsubstanz, welches von $\mathrm{Max} \mathrm{Schultze}$ dahin gedeutet wird, dass die Kerne in wirklichen präformirten Zellenhöhlen liegen. Die Mehrzahl der Kerne zeigt im frischen Zustande und an Osmiumpräparaten keine derartige Zone, sondern liegt einfach in der granulirten Grundsubstanz eingebettet.

Während bei Vergrösserungen, wie die der Fig. 1, die Flächenansicht der electrischen Platte absolut homogen erscheint und keine weitere Structur zeigt, erscheint zuerst bei Objectiven, wie $\mathrm{Hart-}$ n ack's Nr. VII, deutlicher natürlich noch bei Nr. IX sec. (bei welcher Vergrösserung Fig. 2 gezeichnet wurde) an frischen und gut gelungenen Osmiumpräparaten eine über die ganze Fläche der electrischen Platte sich ausdehnende Structur, welche mit Vergrösserungen; wie etwa Hartnack's VII, betrachtet als eine zarte Chagrinirung der electrischen Platte erscheint und von ihrem Entdecker Koelliker als ein zartes Netz markloser Nervenfasern erkannt und als letzte terminale Ausbreitung der Nerven des electrischen Organs gedeutet wurde.

M. Schultze, welcher im Verlauf seiner Untersuchungen über die electrischen Organe der Fische dieses Nervennetz einer genaueren Untersuchung (in Triest) unterwarf, unternahm es, eine Abbildung des Netzwerkes und des Zusammenhanges desselben mit einer stärkeren Nervenfaser zu entwerfen, die vbei Anwendung guter 400500 facher Vergrösserung (Belthle, Schiek) entworfen und etwa dreimal so gross wie das optische Bild gezeichnet wurde, mithin also eine Vorstellung erwecken soll, wie das Netz etwa bei 1500maliger Vergrösserung erscheinen würde. Ich kann mit Hülfe der jetzigen besten Systeme und unter Anwendung der Ueberosmiumsäure, welche M. Schultze bei seinen damaligen Untersuchungen noch nicht benutzen konnte und welche auch hier wunderbar klare Bilder liefert, die Uebereinstimmung der M. Schultze'schen Zeichnung mit der Natur constatiren, so dass M. Schultze's Vertrauen, "dass dereinst die Richtigkeit der Zeichnung bestätigt werde», ein durchaus gerecht- 
fertigtes gewesen ist: Besonders gilt dies von der Darstellung des Ueberganges der Nervenfaser in das Terminalnetz, der in Koelliker's Zeichnung nur in sehr ungenügender Weise wiedergegeben war und welchen besser zu sehen, als ihn M. Schultze bereits vor 15 Jahren gesehen hat, mir auch mit unseren jetzigen so vervollkommneten optischen Iustrumenten nicht möglich gewesen ist. Nur das eine möchte ich in Bezug auf die Abbildung M. Schultze's hervorheben, dass die Maschenräume niemals so quadratisch erscheinen, wie M. Schultze sie gezeichnet, sondern durchweg verzogene und unregelmässige Rhomben mit spitzen und stumpfen Winkeln darstellen; auch sind die Balken des Netzes dicker und die Maschenräume im Verhältniss kleiner, als M. Schultze sie gezeichnet hat. Die Balken sind ganz allgemein ebenso breit, wie der schmale Durchmesser der rhombischen Maschen, so dass die ganze Bildung eine hole Aehnlichkeit mit gewissen Formen der sogenannten gefensterten Häute der Arterienwandungen zeigt, Netzen, in denen Substanz und Lücken ungefähr den gleichen Raum einnehmen.

Diese beiden an der Idealabbildung M. Schultze's zu rügenden Fehler sind in der älteren und bei schwächerer (350facher) Vergrösserung gezeichneten Abbildung Koelliker's vermieden. Hier ist die Flächenansicht der electrischen Platte in der That genau so chagrinirt dargestellt, wie sie bei einer Vergrösserung, wie etwa Hartnack's VII und etwas darunter, erscheint, nicht regelmässig quadratisch gefeldert, sondern unregelmässig gefleckt. Auch ist die Chagrinirung insofern naturgetreu gehalten, als die hellen Maschenräume und die dunklen, dieselben umgränzenden Netzbalken auch die gleiche Breite zu besitzen scheinen.

Gänzlich ungenügend ist die von de Sanctis gegebene bildliche Darstellung des Terminalnetzes. Auch leidet die von demselben Forscher gegebene Beschreibung dieses Netzes an einem eigenthümlichen Widerspruch: er rügt einerseits an der M. Schultze'schen Zeichnung, dass die Maschen quadratisch gezeichnet sind, während sie doch rhombisch sein müssten, - was richtig ist; als einen zweiten Fehler der M. Schultze'schen Abbildung bezeichnet er, dass die Balken im Verhältnisse zu den Maschenräumen viel zu dick erscheinen, während dieselben viel feiner dargestellt werden müssten, was absolut falsch ist.

Nur mit Zagen wende ich mich nach diesen kritischen Erörterungen zu der Erläuterung meiner eigenen Abbildungen zurück, in denen 
ich eine naturgetreue Vorstellung von der hier vorliegenden Structur zu geben versucht habe. Niemals ist mir die alte Wahrheit, dass Kritisiren leichter sei als Bessermachen, so schwer auf das Herz gefallen, wie bei der Anfertigung der vorliegenden Zeichnungen (Figg. 2. 3. 4. 5. 7). Erst nach vielfachen vergeblichen Versuchen, zahllosen angefangenen und liegen gelassenen Zeichnungen sind endlich diese Bilder zu Stande gekommen, von denen ich selbst vielleicht am besten beurtheilen kann, wie wenig sie mit ihren steifen Strichen dem weichen Schwunge und den zarten Linien der Natur entsprechen und wie viel, um mit Lessing zu reden, auf dem langen Wege aus dem Auge durch den Arm in den zeichnenden Stift verloren gegangen ist.

Zu Fig. 2 ist das Koelliker'sche Terminalnetz nur etwa auf einem Fünftel der Fläche der electrischen Platte durchgeführt worden und zwar so, wie es bei einer Vergrösserung von Hartnack VIII oder IX sec. erscheint. Es ist diese Abbildung bestimmt, einen Eindruck zu geben, von der vollständigen Continuität, mit welcher das Nervennetz die Bauchfläche der electrischen Platte überzieht und zu zeigen, wie dasselbe auch unmittelbar unter den hirschgeweihähnlichen Verästelungen, aus denen es sich entwickelt, noch vorhanden ist, - ein Umstand, auf welchen schon M. Schultze gegenüber $\mathrm{K}$ oelliker aufmerksam gemacht hat.

Fig. 3 stellt bei stärkerer Vergrösserung den Uebergang einer feinen Nervenfaser in das Terminalnetz und einen grösseren Bezirk des letzteren dar. Obwohl in der Form der Maschen wie in den relativen Dimensionen rler Maschenräume und der Balken auf das Genaueste den wirklichen Verhältnissen entsprechend, vermag das Bild von der Zartheit und Eleganz der hier vorliegenden Structur doch nur eine kümmerliche Vorstellung zu erwecken.

So viel von dem Koelliker'schen Terminalnetz, welches bis in die neueste Zeit als die letzte Endigung der Nerven im electrischen Organ, sei es definitiv (Ko elliker), sei es als Vorbereitung des Ueberganges in die granulirte Substanz der electrischen Platte selber (M. Schultze) angesehen wurde. Das von mir entdeckte Structurverhältniss der electrischen Platte liegt unmittelbar unter diesem Telminalnetz, d. h. betrachtet man (natürlich mit Immersionslinsen) eine electrische Platte von der Bauchfläche und hat allmählig von oben her durch die gröberen Verästelungen der Nerven hindurch das Terminalnetz in den Focus gestellt, so genügt eine minimale Drehung 
der Mikrometerschraube, das Terminalnetz verschwinden zu machen und an die Stelle desselben die von mir entdeckte Structur treten zu lassen. Es besteht diese Structur in einer vollkommen regelmässigen und gleichartigen Punktirung; die ganze Fläche der electrischen Platte erscheint in fast gleichen Abständen von feinen runden Punkten durchsetzt, die im frischen Präparat glänzend, im Osmiumpräparat dunkelgefärbt erscheinen. Man kann diese vollkommen regelmässige Punktirung lange völlig übersehen und unbeachtet lassen. Hat man sie aber einmal gesehen, so wird man in jedem neuen Präparate auf's Neue ïber die vollkommene Regelmässigkeit und Schönheit dieser Bildung, der ich aus dem ganzen Gebiet der Histiologie kein Analogon an die Seite zu setzen weiss, erstaunen. Wie gesagt, ich hatte schon Tage lang die electrischen Platten mit Immersionslinsen untersucht, ohne auf diese Structur aufmerksam zu werden, die mir plötzlich wie eine Offenbarung in das Auge fiel. Seitdem habe ich das herrliche Bild in keinem frischen und in keinem Osmiumpräparat vermisst. Mit irgend welcher anderen Granulirung der thierischen Gewebe z. B. mit der Granulirung des Protoplasma und dem granulirten Aussehen der molecularen Masse der Hirnrinde ist die vorliegende Structur in keiner Weise zu verwechseln. Dies zeigt sich am klarsten, wenn man durch eine weitere Drehung der Schraube in derselben Richtung wie vorber nun auch das Bild dieser Structur zum Verschwinden bringt und dafür das Bild tieferer (dem Rücken näherer) Schichten der electrischen Platte einstellt, in denen diese Structur nicht mehr vorhanden ist und, welche granulirt im gewönlichen Sinne, d. h. von eben noch messbaren bis zu unmessbaren Körnchen durchsetzt erscheinen. Im Gegensatz zu diesem Bilde erscheint die unmittelbar unter dem Terminalnetz gelegene Schicht der electrischen Platte als eine an und für sich ganz homogene, klare und structurlose helle Grundsubstanz, in welcher in ungefähr gleichmässigen Abständen die ganz identischen glänzenden Punkte eingebettet liegen, so dass mitunter die Flächenansicht einer homogenen von feinen Poren durchsetzten structurlosen Membran vorgetäuscht wird.

Die Anordnung der dunkeln Punkte, die im Allgemeinen durch gleichmässige Zwischenräume getrennt sind, scheint auf den ersten Blick eines bestimmten Princips zu entbehren, und es vergingen einige Tage, ehe ich das in. dieser scheinbar ganz gleichmässigen Vertheilung der Punkte waltende Gesetz auffinden konnte. Der. 
Grund hiervon lag darin, dass ich die erste Bekanntschaft dieses Netzes an solchen Osmiumpräparaten machte, an denen das Koelliker'sche Terminalnetz nur unvollkommen und schlecht zu sehen war. Als ich mich aber dem Studium frischer und der Untersuchung besser conservirter Osmiumpräparate, an denen das Terminalnetz erhalten war, zuwandte, wurde ich alsbald auf eine höchst interessante Beziehung meiner Punkte zu dem Terminalnetz aufmerksam. Um es kurz zu sagen: die Anordnung der Punkte reproducirt getreu die Configuration des über der punktirten Schicht gelegenen Terminalnetzes, so dass die Punkte den Balken des Netzes folgen und den Verlauf derselben nachahmen. Und zwar geschieht dies in der Weise, dass den einzelnen Netzbalken in der punktirten Schicht nicht eine einzelne Reihe oder Zeile von Punkten, sondern mehrere, meist 2 oder gar 3, unregelmässig gestellte Reihen von Punkten entsprechen. Die beiden Figg. 4 und 5 mögen besser wie jede Beschreibung das einschlagende Verhältniss erlältern. In Fig. 4 ist ein Stïck des Koelliker'schen Terminalnetzes dargestellt, in Fig. 5 die unter diesem Terminalnetz gelegene und die Configuration desselben getreu reproducirende Anordnung der Punkte in der punktirten Schicht. Ganz ausnahmslos schliesst sich wie in diesem gezeichneten Präparat so in allen electrischen Platten die Anordnung der in unregelmässigen Doppelreihen angeordneten Punkte dem Ko elliker'schen Terminalnetz an, so dass an den Präparaten, wo das letztere erhalten ist, die Punktreihen genau die Netzform wiederholen. Hat man an diesen Präparaten diese Uebereinstimmung erst sehen gelernt und sich durch wiederholte Beobachtungen, Drehen und Zurückdrehen der Mikrometerschraube, erst überzeugt, dass das Terminalnetz und die Pünktchenreihen ${ }^{4}$ ) sich deckende Bildungen darstellen, so gelingt es dann auch, an solchen Osmiumpräparaten, an denen das Terminalnetz nur undeutlich oder gar nicht zu sehen ist, sich die gleiche Ueberzeugung von der netzartigen Anordnung der zuerst scheinbar gleichmässig durch die homogene, klare Schicht verstreuten dunkeln Körnchen zu verschaffen.

Auf Grund dieser Bilder gelangte ich gleich beim Beginn meiner

1) Zwischen den beiden Bildern, dem des Koelliker'schen Terminalnetzes und dem der Pünktchenreihe erscheint beim Drehen der Mikrometerschraube stets noch ein drittes Uebergangsbild, in welchem beide Structaren sichtbar sind, aber weil sie beide in einander verschwimmen, nur undeutlich erscheinen. 
Untersuchungen zu folgender Vorstellung über den Zusammenhang beider Bildungen, des Terminalnetzes und der von mir entdeckten Pünktchenreihen. Die einzelnen Balken des flächenhaft die Bauchseite der electrischen Platte überziehenden Terminalnetzes tragen an ihrer unteren (d. h. dem Rücken des Thieres zugekehrten Seite) ein System zarter stiftförmiger Fäserchen, welche senkrecht in die Substanz der electrischen Platte eindringen und somit alle frei aufhören. Ich deutete mithin die Pünktchen als Querschnitte feinster Fäserchen.

War diese Voraussetzung richtig, so müssen dieselben durch die Querschnittsbilder bestätigt werden. Es müsste auf dem Querschnitt der der Bauchfläche (dem Terminalnetz) zunächst gelegene Theil der Platte eine feine Längsstreifung senkrecht auf die Ebene der Platte zeigen. In der That schien es, als ob die Thatsachen diese meine Voraussetzungen bestätigen wollten. Das Querschnittsbild der electrischen Platte (Fig. 6), welches viel besser wie an künstlichen Durchschnitten an gefalteten Rändern der einzelnen isolirten Platten untersucht wird, erscheint constant in seinem oberen der Bauchfläche zugekehrten Abschnitt von feinen Längslinien durchzogen, ähnlich dem gestreiften Cuticularsaum der Dünndarmepithelien oder jener feinsten Streifung, die ich an den Cuticularsäumen vieler Cylinderepithelien der Mollusken beschrieben und abgebildet habe ${ }^{1}$ ).

Schon Remak ${ }^{2}$ ) scheint diese Streifung des der Bauchfläche zugekehrten Abschnittes der electrischen Platte gesehen zu habenWenigstens schreibt er den Falten der electrischen Platten den Anschein zu, wals wenn feine Cylinderchen die Dicke des Blättchens bis zur glashellen Membran (welche Remak als die Grundlage der Nervenausbreitung betrachtet) durclisetzen « und spricht von einer "pallisadenähnlichen Stellung feiner Stäbchen nach der Dicke des Blättchens", bei »deren Wahrnehmung ihn seine Mikroskope beinahe schon im Stiche lassen". Bedenklich ist mir bei diesen Angaben Remak's nur der Umstand, dass er diesen gestreiften Saum auch an conservirten Präparaten findet, wo es mir niemals - mit Ausnahme natürlich der Osmiumpräparate - ihn zu sehen gelungen

1) Beiträge zur vergleichenden Histiologie des Molluskentypus. Bonn 1869. S. 42, Figg, 30. 31. 34. 35.

2) Ueber die Enden der Nerven im electrischen Organ des Zitterrochen. Müller's Archiv 1856, S. 470. 
ist. Auch wäre vielleicht die Frage aufzuwerfen, ob die damaligen Mikroskope überhaupt die Wahrnehmung einer so feinen Structur gestatteten ${ }^{1}$ ). Wenigstens konnten weder Koelliker noch $\mathrm{Max}$ Schultze, welcher an erhärteten Präparaten früher etwas derart zu erkennen geglaubt hatte ${ }^{2}$ ), an frischen Präparaten das von $R e-$ mak beschriebene Streifensystem wiederfinden. Auch Ciaccio und de Sanctis erwähnen dasselbe mit keiner Silbe.

Die von mir gegebene Abbildung des Querschnittes der electrischen Platte, so wie derselbe in allen frischen und mit Osmiumsäure behandelten Präparaten ausnahmslos sich darstellt, lehrt zunächst, dass das Terminalnetz einfach und ganz glatt an die Bauchtäche der electrischen Platte angepasst ist, und dass demselben eine irgendwie erheblichere Tiefenausdehnung in keiner Weise zukommt, was der etwas unregelmässige sehr schmale doppeltcontourirte Saum, der den einzigen optischen Ausdruck des querdurchschnittenen Terminalnetzes bildet, zur Evidenz beweist. Ferner wird durch meine Querschnittsbilder die Thatsache zur Gewissheit erhoben, dass die electrische Platte nicht homogen ist, sondern zwei deutlich geschiedene Schichten besitzt, von denen die obere (der Bauchfläche zugekehrte) in ihrer ganzen Ausdehnung feingestreift erscheint, während die untere (der Rückenfläche zugekehrte) keine Spur dieser Streifung zeigt. Diese beiden Schichten sind an Dicke sehr verschieden und zwar beträgt ganz constant die feinstreifige Schicht (nebst dem schmalen doppelten Contour, der den Ausdruck des querdurchschnittenen Terminalnetzes darstellt,) ein Sechstel der Dicke der ganzen electrischen Platte $^{3}$ ). Die übrigen fünf Sechstel des Plattenquerschnitts zeigen

1) Gleichzeitig mit dieser Veröffentlichung Remak's geschah die EnLdeckung und spielte die Controverse über die Streifung der Dünndarmepithelien als ein Structurverhältniss, welches damals die Gränze des optischen Vermögens der Mikroskope bezeichnete. Diese Streifung der Dünndarmepithelien ist - verglichen mit der Streifung in den electrischen Platten ein verhältnissmässig grobes Object, welches jetzt von Mikroskopen zweiten Ranges mit Leichtigkeit demonstrirt werden kann; während die Demonstration der Streifung in den electrischen Platten selbst mit Hartnack'schen Immersionslinsen zu den schwierigen Aufgaben gehört.

2) Sitzungsber. der naturforsch. Gesellschaft zu Halle a. d. S. November 1857 .

3) Ich benutze diese Gelegenheit, hier einige mikrometrisch gewonnene Maassangaben aus der mikroskopischen Anatomie der electrischen Organe und 
ausser einer ausserordentlich feinen Granulirung keine weitere Structur. In dieser untern (der Rückenfläche zugekehrten) granulirten Schicht sind die grossen doppeltcontourirten Kerne eingebettet, welche in unregelmässiger Vertheilung die Substanz der electrischen Platte durchsetzen und deren bei der Beschreibung des Flächenbildes bereits zur Genüge gedacht ist. Die Kerne besitzen ziemlich genau den gleichen Durchmesser wie die electrische Platte selbst und es müssen daher an denjenigen Stellen, wo die Kerne liegen, die begrenzenden Contouren der electrischen Platte etwas mehr wie gewöhnlich auseinanderweichen, um die Kerne zwischen sich fassen zu können. So zeigt die electrische Platte an den kernhaltigen Stellen buckelartige Hervorragungen, und zwar muss ich es nach meinen Präparaten als Regel ansehen, dass, wie auch in Fig. 6 gezeichnet ist, die untere, freie (Rücken-)Seite ganz glatt und eben verläuft und nur allein die Bauchfläche der Platte durch den Kern hervorgewölbt wird. An solchen buckelartig hervorragenden Stellen setzt sich stets auf das Deutlichste die feine Streifung des oberen Sechstels fort und nimmt gewöhnlich den ganzen freien Raum zwischen dem oberen Gränzcontour der Platten und dem Contour des Kernes ein.

Diese Ansicht von der Zusammensetzung der electrischen Platte aus zwei Schichten ist die ursprüngliche ältere Anschauung. Remak,

zwar aus zahlreichen Messungen gewonnene Mittel anzuschliessen. Es betragen :

1) die Dicke der electrischen Platte $=0,0096 \mathrm{~mm}$.,

2) die Dicke des gestreiften Abschnittes $1 / 6=0,0016 \mathrm{~mm}$.,

3) die Dicke des ungestreiften Abschnittes $5 / 6=0,0080 \mathrm{~mm}$.,

4) die Breite der Fasern des Terminalnetzes $=0,0014 \mathrm{~mm}$.,

5) die Distanz der Punkte $=0,00066 \mathrm{~mm}$.

Es beziehen sich diese Zahlenangaben auf nahezu ausgewachsene Exemplarc der Torpedo narke Arist. von durchschnittlich $35 \mathrm{~cm}$. Länge. Bei einem jüngern Individuum von etwa $10 \mathrm{~cm}$. Länge fand ich die dritte der electrischen Platten fast nur halb so gross $(=0,0054)$. Diese Dimensionsverminderung bezog sich allein auf den ungestreiften Abschnitt der Platte $(=0,0028)$, während der gestreifte Abschnitt die unverminderte Dicke von $0,0016 \mathrm{~mm}$. zeigte, folglich etwas über ein Viertel der ganzen Plattendicke einnahm. Auch wollte es mir scheinen, als ob an diesem jungen Exemplar die Punktirung etwas gröber sei wie bei ausgewachsenen Individuen: wenigstens vermochte ich - was mir bisher an ausgewachsenen Exemplaren unmöglich gewesen war - die feine Streifung der gefärbten Ränder an diesen Präparaten bereits mit Hartnack's IX sec. aufzulösen. 
welcher zuerst die rauhe und die glatte Seite der electrischen Plättchen unterschied, fasste die electrische Platte überhaupt als aus zwei Schichten bestehend auf, einer dem Rücken zugekehrten glashellen, kernhaltigen Membran und einer der Bauchseite entsprechenden kernlosen Nervenschicht, eine Ansicht, welche von Koelliker und auch von $\mathrm{Ciaccio}{ }^{1}$ ) getheilt wird. Alle diese Autoren stützen sich auf die Thatsache, dass beide Schichten sich unter den verschiedenen Behandlungsmethoden, Maceration in Chromsäure, Sublimat, Holzessig, Glycerin (Ciaccio) leicht von einander trennen und als isolirte Membranen darstellen lassen. Diese Thatsache kann auch ich bestätigen. An Osmiumpräparaten lösen sich mit grosser Leichtigkeit die beiden Schichten der electrischen Platte auf grössere Strecken von einander los und können mit Leichtigkeit isolirt der mikroskopischen Untersuchung unterworfen werden.

In Fig. 7 habe ich ein nach dieser Hinsicht besonders instructives Präparat gezeichnet, wie es mir öfter im Verlauf meiner Untersuchungen vorgekommen ist, ein durch Zerzupfen gewonnenes Stück einer electrischen Platte, an dem die Theilung und Abspaltung der beiden Schichten von einander eingetreten ist und zwar so, dass an der einen Seite der Figur nur das obere (der Bauchseite zugekehrte) Nervenblatt der Platte erhalten ist, während das untere fünfmal so dicke (der Rückenfläche zugekehrte) Blatt fehlt, während an der anderen Seite des Präparats nur die letztere Schicht erhalten ist, indem die erstere aus dem Terminalnetz und der punktirten Schicht ${ }^{2}$ ) bestehende Lage sich abgeblättert hat. Besonders charakteristisch ist an diesem Osmiumpräparat die verschiedene Tinction der Schichten durch das Reagens, welche ich in der Zeichnung wiederzugeben Sorge getragen habe. Das mittlere Stück des Präparates, welches beide Blätter der electrischen Platte enthält, ist das dunkelste. Das nächstdunkle ist jedoch nicht das fünfmal dickere Stück des Präparates, welches allein die kernhaltige, der Rückenfläche zugekehrte granulirte Schicht, die nglashelle Membran“ Remak's enthält, sondern

1) Ciaccio begeht dabei freilich den Irrthum, die rundea Kerne in die Substanz der Nervenschicht zu verlegen.

2) Ganz besonders muss ich betonen, dass bei diesem Zerfall der electrischen Platte in zwei Blätter ganz ausnahmslos die punktirte Schicht dem Koelliker'schen Terminalnetz folgt und niemals die Spaltung der electrischen Platte etwa zwischen Terminalnetz und punktirter Schicht stattfindet, was gewiss auf einen sehr innigen Zusammenhang beider Bildungen hindeutet. 
das kernlose Nervenblatt, dessen Dicke nur ein Sechstel der Plattendicke beträgt. Es erhellt aus diesem ganz constant eintretenden Verhältniss, dass die Osmiumsäure zu den in dem Nervenblatte enthaltenen histiologischen Elementartheilen eine viel stärkere Verwandtschaft besitzt wie zu der Substanz des unteren fünfmal so dicken Blattes.

Das Studium der beiden so isolirten Blätter ergiebt nur in Bezug auf die Structur des der Rückenfläche zugekehrten Blattes wesentlich neue Aufschlüsse, die sich nicht auch bereits aus der Untersuchung der electrischen Platten an und für sich hätten entnehmen lassen. Zunächst stellt sich zweifellos die Thatsache heraus, dass die doppeltcontourirten Kerne stets in der Substanz der "homogenen glashellen Membran " Remak's und niemals in der Nervenschicht gelegen sind, wie schon Koelliker richtig angibt. Ferner lässt sich an den so isolirten Membranen eine interessante Thatsache erhärten, die für die histiologische Würdigung dieser Membran eine gewisse Bedeutung beansprucht: In der fein granulirten Substanz des der Rückenfläche zugekehrten Blattes habe ich, wo dasselbe von dem Nervenblatt befreit zu Tage lag, bei sehr starken Vergrösserungen in der Fläche desselben sparsame, sehr feine, blasse geschlängelte Fasern von dem Aussehen gewöhnlicher Bindegewebsfibrillen verlaufen sehen, ein Befund, der darauf hinweist, dass das Rückenblatt doch vielleicht nicht so ganz jeder differenzirten Structur entbehrt, wie man bisher angenommen hat.

Das Bild des isolirten Nervenblattes unterscheidet sich von dem gewöhnlichen Flächenbild der electrischen Platte, welches oben ausführlich genug besprochen worden ist, durch Nichts als durch seine grössere Lichtstärke, da die von dem Spiegel des Mikroskops aufsteigenden Strahlen nicht erst die fein granulirte Schicht zu durchsetzen haben, sondern unmittelbar und ungebrochen auf die punktirte Schicht auftreffen. Doch lässt sich auch an diesen Präparaten über das Verbältniss, welches meiner Meinung nach von nun ab den Kern der Discussion über die Structur des electrischen Organs von Torpedo abgeben wird, das Verhältniss nämlich des Koelliker'schen Terminalnetzes zu meinen Pünktchenreihen nichts Bestimmteres ermitteln. Ursprünglich war ich, wie oben angedeutet wurde, der Ansicht, dass die Punktirung des Flächenbildes der Ausdruck eines Systems zarter stiftförmiger Fäserchen sei, welche senkrecht in die Substanz der electrischen Platte eindringen und deren optische 
Querschnitte natürlich als Punkte erscheinen mussten. Ich hatte mir vorgestellt, dass von der (der Rückenfläche zugekehrten) Unterseite der einzelnen Balken des flächenhaft ausgebreiteten Terminalnetzes feinste kurze, gerade Nervenfasern (wie von den feinsten Ausläufern einer Wurzel die Wurzelhaare) senkrecht zu denselben gerichtet ausgingen, welche eine kurze Strecke in die Substanz der electrischen Platte eindringen und alle hier in gleichem Niveau frei aufhören sollten, vergleichbar etwa den Zähnen einer Egge oder den Nadeln einer Hechel. Abgesehen von physiologischen Gründen ${ }^{1}$ ) glaubte ich diese Ansicht begründen zu können einmal dadurch, dass in der Flächenansicht der electrischen Platte die beiden Bilder der in Reihen angeordneten Pünktchen sowie der Balken des Koelliker'schen Terminalnetzes sich vollständig decken, derart, dass die Punkte nur dort auftreten, wo Nervenfasern existiren und dort fehlen, wo keine Nervenfasern vorhanden sind, was gewiss auf einen innigen Zusammenhang beider Bildungen hinweist; zweitens durch das von mir ausführlich beschriebene Bild des Plattenquerschnittes, welcher stets in seinem oberen (der Bauchfläche zugekehrten) Sechstel fein gestreift erscheint. Ich verdanke es der Kritik Max Schultze's, welcher mich brieflich darauf aufmerksam machte, dass die Streifen der gefalteten Ränder ebenso gut wie durch wirklich vorhandene stiftförmige Fortsätze auch bloss durch den combinirten optischen Effect, durch die Zerstreuungsbilder einer Mehrzahl von in verschiedenen optischen Ebenen gelegenen Pünktchen hervorgerufen werden könnten, dass ich die oben entwickelte Vorstellung von der Natur der von mir entdeckten Punktirung nicht mehr mit derjenigen Zuversicht wie früher ${ }^{2}$ ) zu vertreten wage, sondern es jetzt unentschieden lassen muss, ob die an der unteren (dem Rücken zugekehrten) Fläche der Balken des Nervennetzes in so eigenthümlicher Regelmässigkeit angeordneten Elemente Pünktchen (feinste

1) In diesem Falle, wo jede electrische Platte einen einzigen, der Rückenfäche zugekehrten natürlichen Nervenquerschnitt dargestellt bätte, schien die Möglichkeit gegeben, den Schlag des electrischen Organs allein durch die die Innervation begleitende negative Schwankung des Nervenstroms zu erklären. Wenigstens müsste bei dieser Anordnung im Momente der Innervation die Rückenfläche der electrischen Platte positiv, die Bauchfläche negativ electrisch werden, was in der That der Fall ist.

2) Beiträge zur Physiologie von Torpedo. Reichert's und du BoisReymond's Archiv 1873, S. 101. 
Körnchen) oder ob sie feinste stiftförmige Fäserchen sind, ob sie eine reine Kugelform oder die Form eines kurzen, mit seiner Längsaxe senkrecht auf die Fläche der electrischen Platte gestellten Cylinders besitzen. Auch über die Art und Weise, wie diese Elemente mit den Balken des Koelliker'schen Terminalnetzes in Verbindung stehen, wage ich keine Vermuthung und will auch die transscendentale Frage nicht weiter erörtern, ob diese Elemente win « oder "an" dem Nervennetz, $d . h$. ob sie noch in der Substanz des Nervennetzes selbst oder bereits ausserhalb derselben an der untern Fläche des Nervennetzes gelegen sind. Auf dem engen Raume von $0,0016 \mathrm{~mm}$., um den es sich hier handelt und auf welchem durch eine einzige Drehung der Mikrometerschraube die Bilder des Terminalnetzes und der von mir entdeckten Pünktchenstructur in einander schwimmen, ist für eine motivirte Entscheidung dieser Frage in der That kein Raum. Vielleicht gelingt es dereinst, durch die Untersuchung jüngerer Exemplare von Torpedo ${ }^{1}$ ) unsere Erkenntniss dieser Verhältnisse noch etwas zu vertiefen und über die Bedeutung der in dem Nervenblatt der electrischen Platte vereinigten Structur des Terminalnetzes und der Pünktchenreihen etwas Positives auszumachen ${ }^{2}$ ).

Es bleibt noch übrig, die Frage zu erörtern, welche Bedeutung unter diesen Umständen, wenn wir nicht mehr mit Max Schultze eine Homogenität der electrischen Platten, nicht mehr einen Uebergang der Nerven in die Gesammtsubstanz derselben, sondern eine differenzirte Nervenendigung in dem Nervenblatt der electrischen Platte annehmen, welche Bedeutung dann den feingranulirten unteren fünf Sechsteln der electrischen Platte, der glashellen kernhaltigen Membran Rem ak's zukommt. Bei dem jetzigen Stande unserer Kenntnisse (da wir durch Max Schultze's sorgfältige chemische Untersuchungen wissen, dass die Grundsubstanz derselben wesentlich aus Eiweisskörpern besteht), liegen offenbar nur zwei Möglichkeiten vor: Entweder diese Substanz ist eine kernhaltige Masse zusammengeflossenen Protoplasma's, oder es ist eine amorphe Eiweisssubstanz

1) Vgl. oben S. 115, Anmerkung.

2) Um den Fachgenossen die Möglichkeit eines auf eigene Anschauung basirten Ortheils in dieser schwierigen Frage zu verschaffen, bin ich gern erbötig, solchen, die sich desshalb brieflich an mich wenden wollen, von dem Vorrath meiner Präparate, soweit derselbe reicht, abzugeben. 
ähnlich derjenigen, welche in vielen Provinzen der nervösen Centralorgane das Stützgewebe für die nervösen Elementartheile darstellt. Der oben erwähnte Befund feinster, den Bindegewebsfibrillen ähnlicher, in diese Substanz eingelagerter Fasern würde allerdings, wenn es sich bestätigte, dass diese Fasern wirklich dem Bindegewebe zugehören, zu Gunsten der letzteren Alternaltive sprechen. Dennoch ziehe ich es vor, diese schwierige Frage, die in der Histiologie in den verschiedensten Formen wiederkehrend stets auf das Neue gestellt wird ${ }^{1}$ ), zur Zeit noch nicht zu entscheiden, sondern erst den Ausspruch neuer, entwickelungsgeschichtlicher und vergleichendanatomischer Untersuchungen abzuwarten.

Die Entwickelungsgeschichte wird festzustellen haben, ob die beiden Blätter der electrischen Platte aus einer gemeinsamen Anlage oder aus verschiedenen Anlagen hervorgehen, speciell ob die Histiogenese des unteren Blattes mit der Entwickelung der Bindesubstanzen übereinstimmt oder nicht.

Der vergleichenden Anatomie liegt es. ob, in den electrischen Organen von Malapterurus und Gymnotus mit unseren verbesserten optischen Hülfsmitteln nach einer ähnlichen Structur zu suchen, und wenn eine solche sich finden sollte, ihre Beziehungen einerseits zu den Nerven, andererseits $z \mathfrak{u}$ der Substanz der electrischen Platten festzustellen ${ }^{2}$ ).

B erlin, 9. Juni 1873.

1) Vgl. meine Auseinandersetzungen hierüber dieses Archiv VIII, S. 50. Die Histiologie und Histiogenese der nervösen Centralorgane. Berlin 1873, S. 8. S. 115, Anmerkung.

2) Nachträgliche Anmerkung. Während des Druckes dieser Zeilen untersuchte ich Stücke des electrischen Organs von einem der Berliner Exemplare des Malapterurus electricus vac. Beninensis, welche unmittelbar nach dem Tode des Thieres in eine Chromsäurelösung von $1 / 10 \%$ gethan worden und in' dieser Lösung bis jetzt verblieben waren. Die Untersuchung ergab, dass eine gleiche Structur, eine gleiche Punktirung, wie ich sie an den electrischen Platten von Torpedo beschrieben habe, auch an den electrischen Platten des Malapterurus sich findet, und zwar liegt das merkwürdige Verbältniss vor, dass nicht wie bei Torpedo nur die eine Fläche der electrischen Platte diese Punktirung zeigt, sondern beide (Schwanz- und Kopf-) Flächen der electrischen Platten von Malapterurus erscheinen in identischer Weise punktirt. In kürzester Zeit soll die ausführliche und mit Abbildungen versehene Veröffentlichung dieser Beobachtung erfolgen. 


\section{Erklärung der Abbildungen auf Tafel VIII.}

Die römischen Zahlen zeigen die Nummern der Hartnack'schen Objective, die arabischen die der Oculare an.

Fig. 1, IV, 3. Stück einer electrischen Platte, von der Bauchfläche gesehen und den Verästelungsbezirk einer Nervenfaser erster Ordnung entbaltend.

Fig. 2, IX sec., 2. Stück einer electrischen Platte, von der Bauchfläche gesehen und den Verästelungsbezirk einer Nervenfaser zweiter Ordnung enthaltend. Das Koelliker'sche Terminalnetz ist nur auf einem Fünftel der Figur ausgeführt worden.

Fig. 3, X Immersion, 3. Ein kleines Stück einer electrischen Platte, von der Bauchfläche gesehen, um das Terminalnetz und den Uebergang einer feinsten Nervenfaser in dasselbe zu zeigen.

Fig. 4, X Immersion, 3. Ein kleines Stück des Terminalnetzes.

Fig. 5, X Immersion, 3. Dasselbe Stück der electrischen Platte bei etwas tieferer Einstellung.

Fig. 6, X Immersion, 3. Ein (gefalteter) Querschnitt einer electrischen Platte. Die Bauchfläche ist die obere.

Fig. 7, IX Immersion, 2. Ein Stück einer electrischen Platte, von der Bauchfläche gesehen. Zerzupfungspräparat. In der Mitte sind beide Schichten der electrischen Platte, links nur die Nervenschicht, rechts nur die structurlose Grundlage allein erhalten. 